\title{
МАТРИЦА СТОИМОСТНЫХ ИЗМЕРЕНИЙ НЕОДНОРОДНОГО ЭКОНОМИЧЕСКОГО ПРОСТРАНСТВА ТРАНСАКЦИЙ "
}

\author{
(c) 2020 Самсонов Руслан Александрович \\ кандидат экономических наук, директор \\ Алтайский институт труда и права (филиал) ОУП ВО «Академия труда и социальных отношений», \\ Россия, Барнаул \\ E-mail:SamsonovR@yandex.ru
}

В статье обосновывается необходимость преодоления «субстанционального» подхода в методологии стоимостной оценки и перехода к «целостной» парадигме многомерных стоимостных измерений. На основе гипотезы об относительности стоимостных измерений неоднородного экономического пространства трансакций с учетом положений синтетической концепции ценности как соотношения категорий полезности и стоимости разрабатывается матричная модель стоимостных измерений, позволяющая проявить и исследовать закономерности их строения, содержания и динамики. В заключение ставится проблема, связанная с необходимостью формирования эффективной институциональной инфраструктуры стоимостных измерений.

Ключевые слова: матрица стоимостных измерений, относительность, многомерность, неоднородность экономического пространства, трансакции.

К вопросу стоимостных измерений неоднородного экономического пространства трансакций

В современной оценочной науке доминирует, условно назовем его, «субстанциональный» подход, который ориентирован практически исключительно на исследования источников, содержания, размеров и структуры различных форм стоимости. Классическими примерами являются соотношения категорий общественно необходимых затрат труда и предельной полезности, несколько сглаженные в рамках двухкритериальной модели равновесной стоимости с учетом фактора времени. Институциональные аспекты данного подхода получили разработку в поведенческих концепциях, рассматривающих стоимость как результат сделки (конвенции) между людьми или свойство определенной совокупности правоотношений $[2 ; 3 ; 18]$.

Данная тенденция также проявляется в чисто практической плоскости, в качестве примеров выступают не только множественные виды стоимости, закрепленные в международных и федеральных стандартах финансовой и оценочной деятельности [7; 14], но и вся система счетоводства на микро и макроуровнях [10; 13; 16]. При этом фрагментарность и ограниченность «субстанционального» подхода в понимании «целостной картины» стоимостных оценок уже давно замечены и имеется ряд серьезных исследований, посвященных его критике, попыткам развития и преодоления.

В области макроэкономики отметим работы ученых под руководством Д.Стиглица, А.Сена и Ж-П Фитусси [13] по проблеме совершенствования метрического аппарата стоимостных оценок макроэкономических показателей (что обусловлено их недостатками, связанными с недооценкой социального неравенства, взаимовлияния экономики и окружающей среды, воспроизводства экстерналий [6; 8]) посредством разработки панелей индикаторов и индексов устойчивого развития.

С позиции уровня наноэкономики отдельного человека, выделяются исследования по экономике счастья Р.Истэрлина [16; 19], развивающие теории полезности [1; 17], с учетом гипотез Фридмена-Севеджа и Марковица [15], относительно роли субъективных факторов в оценке ценности объектов экономического пространства. В данных работах выявлена тенденция («парадокс Истэрлина») расхождения корреляции, особенно в долгосрочном периоде, между динамикой доходов стран и уровнем счастья их населения.

В качестве интегрального подхода выступают работы по синтетической концепции ценно-

\footnotetext{
* Статья подготовлена при финансовой поддержке РФФИ в рамках научного проекта № 19-010-01045 А «Исследование относительности стоимостных измерений неоднородного экономического пространства трансакций: микро- и макроэкономические аспекты».
} 
сти [5], в рамках которых категории полезности (проявляется в виде показателей качества) и стоимости (проявляется в виде цен) исследуются с позиции их диалектической взаимообусловленности и особенностей воспроизводства на разных уровнях экономики (рис. 1).

На сущностном уровне экономических отношений, как отмечают представители данной школы [5], соотношение категорий полезности и стоимости образует ценность оцениваемого блага, преодолевая тем самым противоречия разных научных школ и подходов. Причем если стоимости на уровне наноэкономики являются усредненными и условно постоянными («сжатыми») величинами при относительном многообразии и динамизме состояний полезности, то на уровне мини и микроэкономики их роль постепенно выравнивается, а на макро- и мегауровнях, наоборот, стоимостные формы проявляются во всем разнообразии и динамике своих характеристик, но уже в условиях усредненности и однородности параметров полезности.

При этом согласно сформулированной и разрабатываемой нами гипотезы об относительности и многомерности стоимостных измерений неоднородного экономического пространства трансакций [9; 10] в нем одновременно сосуществуют, воспроизводятся и взаимодействуют четыре различных стоимостных измерения, каждое из которых обладает уникальным набором характеристик и принципов институциональной регуляции (рис. 2).

Фундаментальными свойствами экономического пространства в предлагаемой нами мо- дели являются его неоднородность и многомерность стоимостных измерений. Структурным ядром экономического пространства выступает рыночное измерение положительных стоимостей (товарный оборот), с ним взаимодействуют - квазирыночное измерение отрицательных антистоимостей (различные виды ущерба), псевдорыночное измерение отрицательных стоимостей (положительные экстерналии) и антирыночное измерение положительных антистоимостей (отрицательные экстерналии), образующие в условиях пространственной и временной аттракции синергетические эффекты в виде проявления «полной» меновой стоимости трансакций, которую предлагаем определять как экономическую стоимость.

Формула оценки экономической стоимости в самом общем виде может быть записана в форме следующей математической записи [9, с. 262]:

$$
Э \mathrm{C}=\Pi \mathrm{C}-\mathrm{OA}+(\mathrm{OC}-\Pi \mathrm{A})
$$

где ЭС - экономическая стоимость; ПС - положительная стоимость; ОА - отрицательная антистоимость; ОС - отрицательная стоимость, ПА - положительная антистоимость.

Таким образом, при комплексном исследовании вопросов стоимостной оценки различных объектов необходимо учитывать не только «субстанцию» стоимости как таковую, но и ее целостное воспроизводство в многомерных стоимостных измерениях экономического пространства трансакций с учетом его неоднородного и иерархического строения.

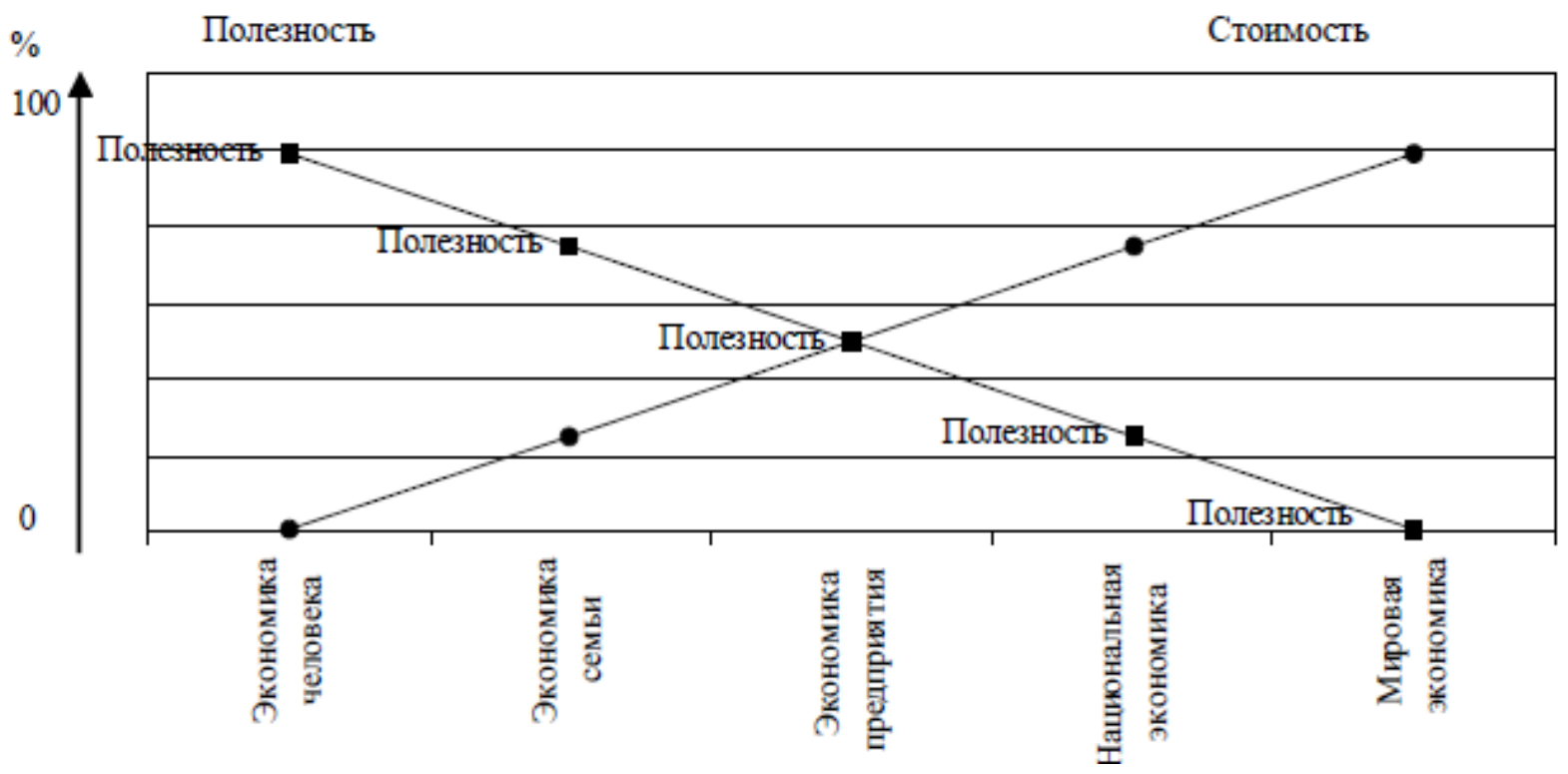

Puc. 1. Доминирование полезности и стоимости блага на разных уровнях хозяйствования [5, с. 18] 


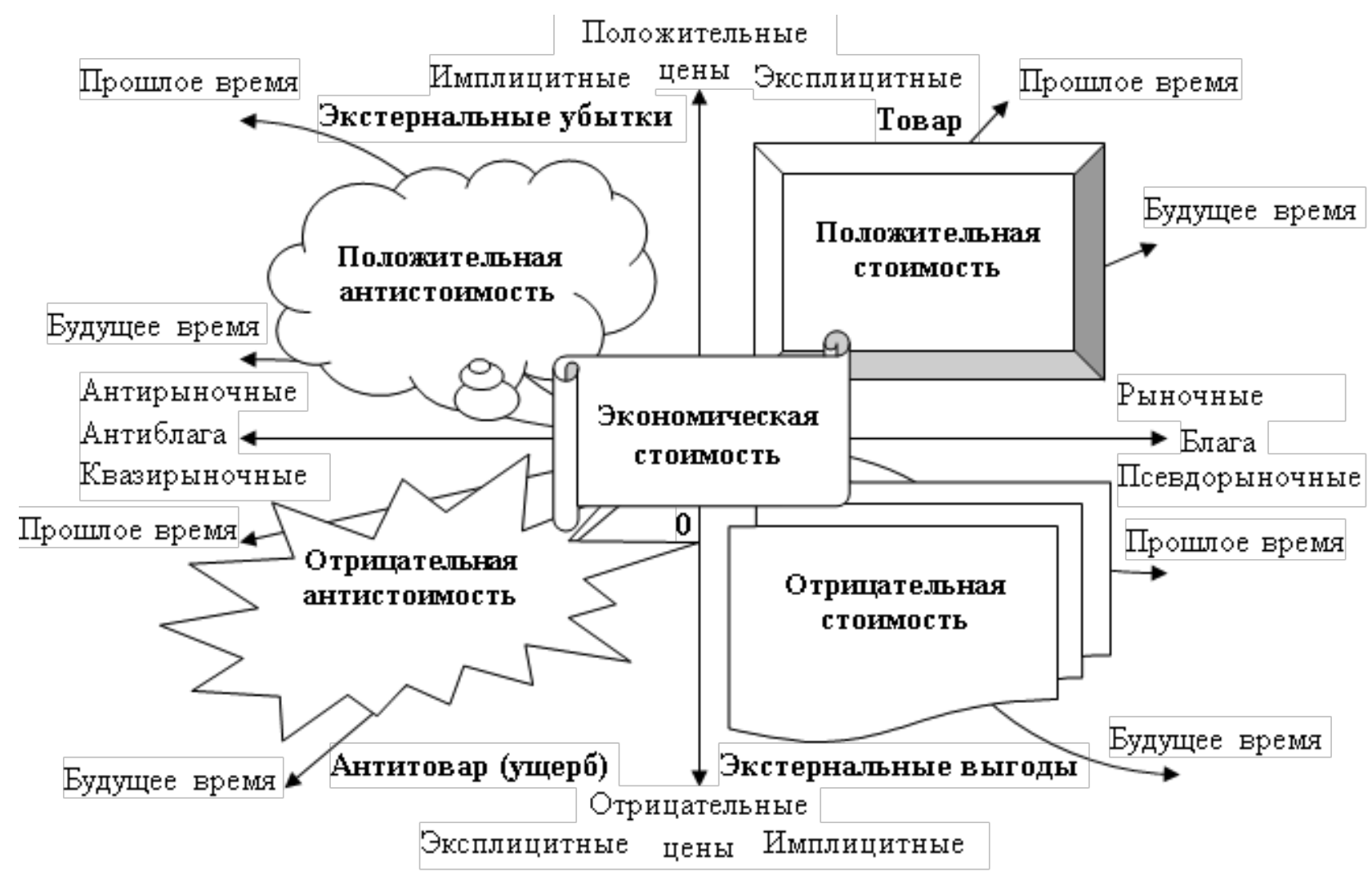

Puc. 2. Модель относительности стоимостных измерений неоднородного экономического пространства трансакций $[9$, с. 264]

Матричная модель стоимостных измерений неоднородного экономического пространства трансакций

Для изучения системы стоимостных измерений в неоднородном экономическом пространстве трансакций предложим объединить «иерархическую» и «метрическую» модели в виде матрицы, что позволит проявить и исследовать закономерности их строения, содержания и динамики, а также уточнить факторы модальности проявления стоимости и полезности.

Представленная матричная модель (табл.) позволяет уточнить, дополнить и детализировать параметры соотношения категорий полезности и стоимости, рассмотренные в синтетической концепции ценности [5], с позиции закономерностей воспроизводства многомерных стоимостных измерений неоднородного экономического пространства трансакций.

На наноуровне экономики человека размерность и динамика стоимости благ, в отличие от разно размерных, неоднородных и изменчивых характеристик их ожидаемой полезности, пребывает в усредненном и относительно устойчи- вом по отношению к отдельному человеку состоянии, который стремится максимизировать функции полезности и минимизировать функции антиполезности при определенных ограничениях. На этом уровне все стоимостные измерения, реализующиеся в простой, единичной (случайной) форме, находятся как бы в «схлопнутом состоянии» (нет условий для развития отношений мены и установления ее общественно необходимых пропорций), будучи сведены к определению стоимости на основе альтернативных издержек упущенных возможностей («экономика Робинзона»). В данном случае «полная» меновая стоимость трансакций $\left(\mathrm{C}_{\Pi}\right)$ редуцирована до имплицитного состояния положительной стоимости (2), находящейся в потенции.

$$
\mathrm{C}_{\Pi} \approx \Pi \mathrm{C}_{\Pi}
$$

На миниуровне экономики семьи при сохранении в целом роли фактора полезности (с учетом некоторого снижения вариативности ожидаемой полезности благ) уже наблюдается возрастающая волатильность интернальных стоимост- 
Таблица. Матрица стоимостных измерений неоднородного экономического пространства трансакций

\begin{tabular}{|c|c|c|c|c|}
\hline \multirow{2}{*}{$\begin{array}{l}\text { Индикаторы } \\
\text { модальности }\end{array}$} & \multicolumn{4}{|c|}{ Стоимостные измерения } \\
\hline & Рыночное & Квазирыночное & Псевдорыночное & Антирыночное \\
\hline \multicolumn{5}{|c|}{ Мегауровень хозяйствования (мировая экономика) } \\
\hline Стоимости & + & + & + & + \\
\hline Полезности & - & - & - & - \\
\hline \multicolumn{5}{|c|}{ Макроуровень хозяйствования (национальная экономика) } \\
\hline Стоимости & + & + & + & + \\
\hline Полезности & + & + & - & - \\
\hline \multicolumn{5}{|c|}{ Микроуровень хозяйствования (экономика предприятия) } \\
\hline Стоимости & + & + & + & + \\
\hline Полезности & + & + & + & + \\
\hline \multicolumn{5}{|c|}{ Миниуровень хозяйствования (экономика семьи) } \\
\hline Стоимости & + & + & - & - \\
\hline Полезности & + & + & + & + \\
\hline \multicolumn{5}{|c|}{ Наноуровень хозяйствования (экономика человека) } \\
\hline Стоимости & - & - & - & - \\
\hline Полезности & + & + & + & + \\
\hline
\end{tabular}

ных измерений, связанных с обращением благ и компенсацией ущербов преимущественно в развернутой и всеобей формах стоимости, что обусловлено нарастанием внутренних меновых трансакций, деятельностью субъектов на так называемых брачных рынках и экономическими условиями биосоциального воспроизводства «человеческого капитала» в домашних хозяйствах. На миниуровне экстернальные стоимостные измерения находятся как бы в «свернутом» между субъектами, предприятиями, отраслями, территориями и периодами времени состоянии.

Таким образом, показатель «полной» меновой стоимости трансакций на уровне миниэкономики представляет разницу между значениями показателей положительной стоимости и отрицательной антистоимости:

$$
\mathrm{C}_{\Pi}=\Pi \mathrm{C}-\mathrm{OA}
$$

На микроуровне экономики предприятия происходит выравнивание роли факторов стоимости и полезности во влиянии на хозяйственные процессы. На данном уровне экономики, наряду с активными интернальными, начинают «раскрываться» и проявляться экстернальные стоимостные измерения, что связано с полной реализацией экономического потенциала отношений мены, выраженных в законченной, денежной форме стоимости, в процессе реализации множества разного рода трансакций между различными субъектами. В данных условиях наблюдается нарастающая рационализация в поведении субъектов мены, что, с позиции функций ожидаемой полезности, обусловливает их преимущественно индифферентное отношение к риску с использованием методов системного анализа и риск-менеджмента.

Формула экономической стоимости на уровне микроэкономики может быть записана в общем виде (4), но с оговоркой, что экстернальные стоимостные измерения проявляются лишь в ближнем хозяйственном окружении предприятия на определенной территории, а внешние макро- и мега эффекты могут зачастую оставаться незамеченными и неучтенными.

$$
\ni C=\Pi C-O A+\left(O C^{* * *}-\Pi A^{* * *}\right)
$$

На макроуровне национальной экономики роль факторов полезности благ стабилизируется и выравнивается, так как функции полезности и антиполезности уже практически реализовались и происходит их агрегирование, приведение к однородному состоянию и в соответствие общественно необходимому уровню (разработке данного вопроса посвящены исследования по теории измерения полезности В.А. Сибирцева [12]). В условиях макроэкономики и нарастания эффектов масштаба значения предельной полезности и по размеру, и по динамике становятся усредненными и несущественными величина- 
ми, которые к тому же, как показатели качества, должны соответствовать «техническим» стандартам (относится к сфере интернальных рыночных и квазирыночных стоимостных измерений). При этом стоимостные измерения предстают уже не просто многомерными, в них начинают проявляться различные динамические и синергетические эффекты в виде систематической ренты [4], которая периодически воспроизводится, меняя лишь точки отраслевой и пространственной локализации, и экономической стоимости, что обусловлено наличием на национальном уровне особого институционального актора - субъектов публичной власти, представляющих и реализующих общественные интересы.

Следует отметить, что экономическая стоимость на макроуровне выражается в общем виде (5), но с учетом ограничений по интернализации издержек и выгод экстернальных стоимостных измерений в контексте воспроизводства и институционального регулирования внешних эффектов в масштабах трансакций мировой экономики.

$$
\ni \mathrm{C}=\Pi \mathrm{C}-\mathrm{OA}+\left(\mathrm{OC}^{*}-\Pi \mathrm{A}^{*}\right)
$$

На мегауровне мировой экономики рассмотренные процессы продолжают усиливаться и получают свое полное проявление. Величины изменения предельной полезности на данном уровне настолько ничтожно малы и однородны, что ими можно пренебречь с позиции индикаторов мировой экономики, в масштабах которой показатели качества также получают международную унификацию и стандартизацию. В межстрановых условиях стоимостные измерения становятся гипердинамичными и многообразными с позиции своего экономического и технологического содержания, что ведет к возникновению новых институциональных форм регуляции стоимостных отношений в виде глобальных цепочек создания добавленной стоимости [11], повсеместное распространение которых сопровождается нарастанием глобальных вызовов и проблем, воспроизводством целого каскада положительных и отрицательных экстерналий.

Как результат, на мегауровне экономическая стоимость приобретает целостную и законченную форму (6).

$$
Э \mathrm{C}=\Pi \mathrm{C}-\mathrm{OA}+(\mathrm{OC}-\Pi \mathrm{A})
$$

На уровне мировой экономики появляется потенциальная возможность интернализации издержек и выгод экстернальных стоимостных измерений на основе международных институциональных механизмов регуляции трансграничных трансакций.

\section{Заключение}

По своей экономической сути, стоимостные отношения мены не исчерпываются только рыночными оценками, так как экономика намного «шире» рыночного измерения трансакций, поэтому необходимо учитывать их «полную» меновую стоимость, которая также заключает в себе квазирыночное измерение отрицательных антистоимостей, псевдорыночное измерение отрицательных стоимостей и антирыночное измерение положительных антистоимостей. Взаимодействуя между собой на разных уровнях хозяйствования различные стоимостные измерения формируют новое системное качество неоднородного экономического пространства трансакций в виде многомерной, синергетической и динамической сущности - экономической стоимости, проявляющейся в виде экономических цен.

Для реализации на практике механизма экономических цен, сначала в целях стоимостной оценки стратегических и социально значимых объектов, необходимо завершить работу по разработке математических моделей оценки экономической стоимости, осуществить эмпирические и статистические верификации выявленных закономерностей, формализовать и закрепить категорию «экономическая стоимость» в федеральных стандартах оценки. Отдельно следует сказать о необходимости выработки эффективных институциональных механизмов перспективной и ретроспективной ответственности, определяющих уровень трансакционных издержек и реализацию прав собственности, что, способствуя интернализации экстерналий, непосредственно влияет на уровень благосостояния общества в долгосрочном периоде времени. 


\section{Библиографический список}

1. Алчиан А. Значение измерения полезности // Вехи экономической мысли. Теория потребительского поведения и спроса Т.1. Под ред. В. М. Гальперина.-СПб.: Экономическая школа, 2000. 380 с.

2. Алчиан А. Стоимость // Вехи экономической мысли. Рынок факторов производства. Т.3. Под ред. В. М. Гальперина.-СПб.: Экономическая школа. 2000. 489 с.

3. Блауг М. Экономическая мысль в ретроспективе.-М.: Дело Лтд, 1994. 720 с.

4. убарь А. И. Систематическая рента // Известия Алтайского государственного университета. 1999. № 2. С. 107-111.

5. Гськова М.Ф., Стерликов П. Ф., Стерликов Ф.Ф. От стоимости к ценности от ценности к стоимости? // Экономические науки. 2014. № 9 (118). С. 17-20.

6. Коуз Р. Фирма, рынок и право.- М.: Новое издательство, 2007. 224 с.

7. Международные стандарты оценки / Международный совет по стандартам оценки; [И.Л.Артеменков (гл. ред.) и др.].- Москва: Российское о-во оценщиков, 2020.

8. Пигу А. С. Экономическая теория благосостояния.- М.: Прогресс. в 2-х томах. 1985.

9. Самсонов Р. А. По ту сторону положительной стоимости...!? Гипотеза об относительности стоимостных измерений экономического пространства // Управление современной организацией: опыт, проблемы и перспективы. - Барнаул: АЗБУКА, 2014. Вып. 6. 368 с. С. 255-265.

10. Самсонов Р.А. Проблемы стоимостных измерений неоднородного экономического пространства трансакций.- Барнаул: Изд-во Алт. ун-та, 2019. 176 с.

11. Самсонов Р.А., Бочаров С. Н. Систематическая рента в глобальных цепочках создания добавленной стоимости // Управленец. 2019. Т. 10. № 6. С. 2-14.

12. Сибирцев В.А. Теория измерения полезности.-М.: Изд-во «ИРИС ГРУПП», 2011. 308 с.

13. Стиглии Дж., Сен А., Фитусси Ж.-П. Неверно оценивая нашу жизнь: Почему ВВП не имеет смысл? Доклад Комиссии по измерению эффективности экономики и социального прогресса.- М.: Изд-во Института Гайдара, 2016. 216 с.

14. Федеральный стандарт оценки «Цель оценки и виды стоимости (ФСО N 2)», утвержденный приказом N 298 Минэкономразвития России от 20.05.2015 г.

15. Фридмен М., Сэвидж Дж. Анализ полезности при выборе среди альтернатив, предполагающих риск // Вехи экономической мысли. Теория потребительского поведения и спроса. Т.1. Под ред. В. М. Гальперина. СПб.: Экономическая школа. 2000. 380 с. С. 208-249.

16. Чинакова Н.В. Экономика счастья: современные исследования и дискуссии // Мир экономики и управления. 2016. Т. 16. № 1. С. 101-115.

17. Шумейкер П. Модель ожидаемой полезности: разновдности, подходы, результаты и пределы возможностей / THESIS, 1994, вып. 5. С. 29-80.

18. Эволюция теории стоимости / Под ред. проф. Я. С. Ядгарова.-М.: ИНФРА-М, 2016. 253 с.

19. Easterlin R.A., McVey L.A., Switek M., Sawangfa O., ZweigJ. S. The happiness-income paradox revisited. Proceedings of the National Academy of Sciences of the United States of America, 2010, vol. 107, no. 52, p. 22463-22468. URL: http://www.pnas.org/content/107/52/22463 (date of access 12.10.2020). 